\title{
An Artificial Intelligence Approach for Predicting Different Types of Stroke
}

\author{
Ankitha $\mathrm{S}$ \\ Department of Computer Science and Engineering \\ Vidyavardhaka College of Engineering \\ Mysore, India \\ Harshavardhan $\mathrm{N}$ \\ Department of Computer Science and Engineering \\ Vidyavardhaka College of Engineering \\ Mysore, India
}

\author{
Jahnavi V \\ Department of Computer Science and Engineering \\ Vidyavardhaka College of Engineering \\ Mysore, India \\ Deepthi M \\ Department of Computer Science and Engineering \\ Vidyavardhaka College of Engineering \\ Mysore, India
}

\author{
Remanth M \\ Department of Computer Science and Engineering \\ Vidyavardhaka College of Engineering \\ Mysore, India
}

\begin{abstract}
Stroke is a second leading cause of death and they have been serious, long-term incapacity. Stroke is the sudden demise of cerebrum cells because of a nonattendance of oxygen, caused by blockage of bloodstream or break of a supply route to the mind. According to World Health Organization in the upcoming year's stroke will continue growth death rate. The many works have been carried out for detecting stroke diseases. An artificial intelligence solution which uses deep learning to predict stroke and its forms. Examples are an ischemic stroke, a bleeding stroke, a transient ischemic attack. The collection of data from the medical institute in our work. The system of pre-processing expels archives of copies, incomplete data and conflicting information. Principle element analysis algorithm is measurement is used to minimize predictions and profound learning by determining whether or not the person is experiencing stroke disease. This changes the classification by deep learning in order to predict the stroke condition. When the patient details are entered, it checks with trained model and forecasting of different types of stroke. It this work mainly focuses better way for predicting stroke and different type of stroke.
\end{abstract}

Keywords - Artificial intelligence, artificial neural network and back propagation algorithm.

\section{INTRODUCTION}

Stroke is a third imperative death and long-term incapacity cause. Stroke is a psychological injury that happens at any moment. A coagulation happens in veins or blood channel split. As indicated by the stroke of the world health organization in the coming years will continue to increase the rate of death, so serious treatment of the condition must be swift as expected under the circumstances. Overall, a million people suffer a stroke. Inability requires confrontational deformity, vision loss, loss of balance, and speech. Stroke is a major unsafe condition that hurts the cerebrum, like a heart strike that hurts the heart. It does not pump blood and provide oxygen to the cells of the brain. Everyone is overcoming with a certain danger of stroke. The stroke may cause loss of motion, sudden chest pain, speech impotence, loss of memory and limits of thought, dim like state, or death. Stroke affects the adult of all ages. It can be managed by useful regulation and it is important to adjust risk factors. The report says that the most known remedial mistake exists in terms of drugs expiry, off-kilter medicines, mistaken assumptions and treatment given to the wrong patient.

Stroke for the most part three composers:

- Ischemic stroke

- Hemorrhagic stroke

- Transient ischemic stroke

\section{METHODOLOGY}

In fitting algorithm to train the set, it is difficult to find a good or even a well-performing machine learning algorithm for a particular dataset. We went through a process of trial and error to settle on a short list of algorithms that provides better result. We studied a couple of algorithms. In our work we are going to show and discuss the performance of Naive Bayes algorithm and Back propagation algorithms. 


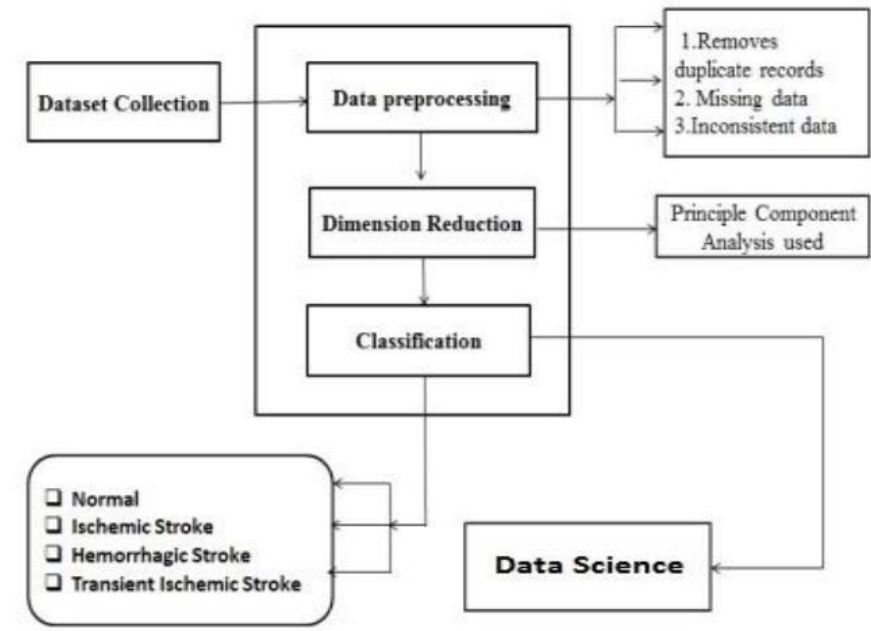

Fig: System Architecture

The Machine Learning system uses the training data to train models to see patterns, and the test data are used to assess the trained model's predictive performance. Classification is regarded as an example of supervised learning in machine learning jargon, learning where a training set of properly described observations is available. We use Naive Bayes classifier, Random forest and the KNN algorithm or Decision tree algorithm is used to predict and classify the different types of strokes According to the stroke robotic research investigations and its subtypes were on the picture methods and CT scanning and MRI. An artificial neural system provides a general approach to the problems. It receives neural system order calculation to predict the stroke infection. A stroke disease based Artificial neural system enhances analytical accuracy with higher consistency. Here the proposed system is an automation for stroke prediction using machine learning or AI techniques. $\mathrm{s}$

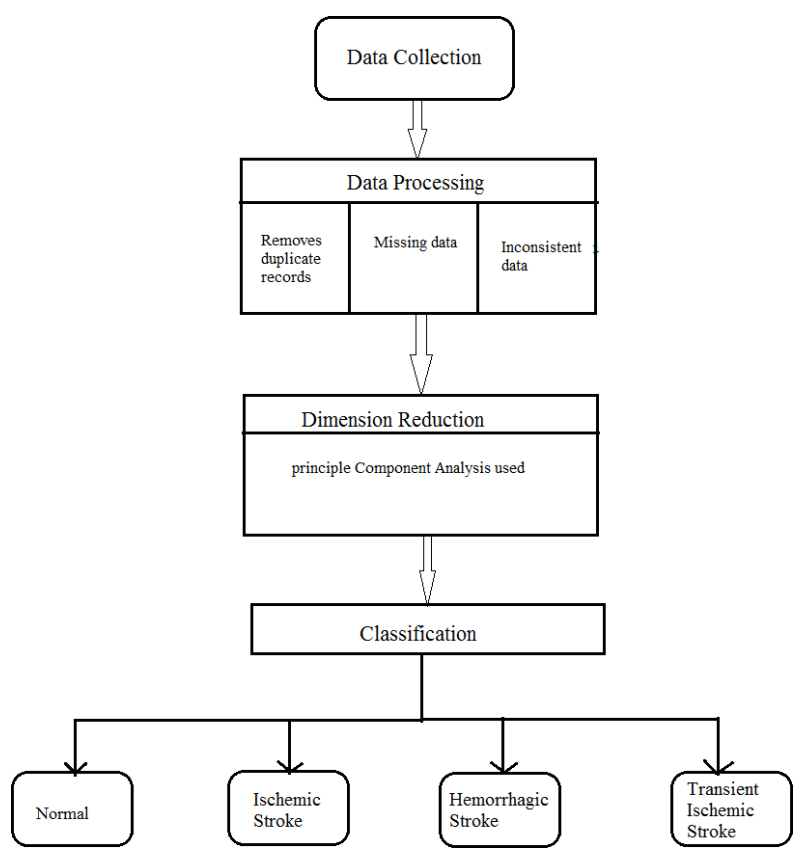

Fig: FlowChart

\section{RELATED WORK}

Healthcare companies also deliver many advantages such as fraud detection in healthcare insurance, accessibility of medical facilities for patients at cheap prices, recognition of better treatment methodologies, developing innovative healthcare policies, efficient hospital resource management, enhanced customer relationships, improved patient care and hospital infection control. Detection of stroke form is also one of the major areas of medical research. There is no automation for Stroke disease prediction.

[1] In this paper author have used readily available data tools such as Rapid Miner tool for implementation. But there is some limitations using tools like Rapid Miner and other tools namely R-Tool, Wekaa tool where the results can be easily obtained but the testing of these is not possible. Here the author used small dataset for the prediction of stroke which results in less accurate results.

[2] The Random Forest and Support Vector Machine (SVM) were used for implementation in this paper writer. The main objective of this paper is to compare different approaches to machine learning with model classification and risk prediction. but using $\mathrm{KNN}$ and SVM techniques produces graphical outputs where the distinguishing will be difficult in the graphical method.

[3] In this paper the data set used by the author for implementation is only static data available on the UCI machine learning repository. Therefore there are certain limitations in this paper such as it uses data mining techniques, requires huge data and more time is required for the prediction of the strokes.

[4] In this paper their approach for the prediction of stroke probability the author have used the fuzzy congnitive maps and non-linear hebbian learning algorithm. Since the user have make use of fuzzy congnitive maps where the output results will have less accuracy. And the prediction of Stroke probability will lead to less accurate results.

[5] The author has compared the three techniques in this paper and they are the deep learning technique, Naïve Bayes and the Stroke disease prediction support vector machine. It requires more time for prediction by using all these techniques, and it also requires huge data.

[6] The author's goal in this paper is to suggest an ischemic stroke detection system using computer-aided diagnostic technology based on an unsupervised method of enhancing perception of features to identify ischemic stroke areas in brain CT images.

[7] The author has proposed a counter-lateral symmetry inorder algorithm in this paper to detect the stroke. This algorithm's main feature is to identify the different types 
of strokes such as acute, chronic offenses and hemorrhages. The algorithm is used here to detect the slices affected by the stroke in a volume of CT.

\section{CONCLUSION}

Stroke Type prediction has become a global health issue and is an area of concern. Current system is a manual, time consuming, requires more experience of doctor and expensive. Most investigations performed on the robotized analysis of stroke and its subtypes were on the picture preparing methods and CT scan and MRI. An artificial neural system gives a general method for moving toward issues. To predict the stroke infection, it receives order calculation neural system. An Artificial neural system based expectation of stroke illness enhances the analytic exactness with higher consistency. Proposed system is an automation for stoke prediction using machine learning or AI techniques.

\section{ACKNOWLEDMENT}

The authors express their appreciation for the help given by our mentors and faculty members who have guided us throughout the research and helped us achieve desired results in a given time.

\section{REFERENCES}

[1] Jia Xie, Zhu Wang,Zhiwen Yu, Bin Guo, "Enabling Efficient Stroke Prediction by Exploring Sleep Related Features.

[2] Luis Garcia-Temza, Jose L. Risco-Martin, Jose L. Ayala, Gemma Reig Rosello, Juan M. Camarasltas, "Comparison of Different Machine Learning Approaches to Model Subtype Classification and Risk Prediction.

[3] SFariborz Khorvash, Rasul Norouzi, "Prediction and Control of Stroke by DataMining".

[4] Mahsa Khodadadi, Heidarali Shayanfar, Kevian Maghooli and Amir Hooshang Mazinan, "Prediction of Stroke Probability Occurrence Based on Fuzzy Cognitive Maps".

[5] Pattanapong Chantamit-o-pas and Madhu Goyal, "Prediction of Stroke Disease using DeepLearning Model".

[6] Yeu-Sheng Tyan, Ming-Chi Wu, Chiun-Li Chin, Yu-Liang Kuo, Ming-Sian Lee and Hao-Yan Chang, "Ischemic Stroke Detection System With a Computer-Aided Diagnostic Ability Using an Unsupervised Feature Perception Enhancement Method".

[7] Mayank Chawla, Saurabh Sharma, Jayanthi Sivaswamy, Kishore L.T, "A method for automatic detection and classification of storke from brain CT iamges".

[8] D. Cosic and S. Loncaric, "Computer system for quantitative analysisof ich from ct head images," in 19th Annual International Conferenceof the IEEE, 1997.

[9] K. Sklinda, P. Bargiel, A. Przelaskowski, T. Bulski, J. Walecki, andP. Grieb, "Multiscale extraction of hypodensity in hyperacute stroke,'Med Sci Monit, 2007.

[10] C.-C. Liao, F. Xiao, J.-M. Wong, and I.-J. Chi-ang. A knowledge discovery approach to di-agnosing intracranial hematomas on brain ct:recognition, measurement and classification. InMedical Biometrics, pages 73-82. Springer, 2007 